\title{
A Model of Active Roll Vehicle Suspension
}

\author{
I. Cech
}

\begin{abstract}
This paper describes active suspension with active roll for four-wheel vehicle (bus) by means of an in-series pump actuator with doubled hydropneumatic springs. It also gives full control law with no sky-craping. Lateral stiffness and solid axle geometry in the mechanical model are not neglected. Responses to lateral input as well as responses to statistical unevennesses show considerable improvement of passengers comfort and safety when cornering.
\end{abstract}

Keywords: active suspension, roll-yaw model of a four-wheel vehicle, cross control, solid axle, hydraulic control, pump actuator.

\section{Introduction}

A mathematical description of a model of active roll vehicle suspension is made in steady state of the harmonic input in symbolic form. The complex amplitudes and effective values are denoted by capital letters and the instantaneous values, and also the constant values, are denoted in lower-case letters. Differentiations are substituted by the operator $\mathbf{s}=i 2 \pi f$, second differentiations by $\mathbf{s}^{2}$, and so on. As our model includes two more stages of differentiations than in usual mechanical models, a complex symbolic form is necessary to handle the model.

Only linear relations are used.

A complete 4-wheel vehicle is dealt with here, but no heave input is presumed.

The parameters relate to a tall bus.

The computed answers are compared with passive suspension.

The results are given for both vertical and horizontal input, in spectral form and in impulse form.

Unlike other proposed solutions, e.g. $[1,2,3]$ our project includes the static load control, and should be low-powered. This is because of the in-series character of the control, so that static displacement compensation takes no power. In addition, the controller uses no throttling and works against no static load. The control scheme is simple, so that there are no problems with stability.

There is also the possibility of achieving active suspension using a source of force parallel to air suspension (which includes the static load displacement control). This source of force can be implemented by a linear electric motor. However, the power produced by the linear motor is small in comparison with a rotational electric motor with gearing.

\section{Mechanical model}

Dealing only with linear relations, we first presume the unevennesses decomposition diagram in Fig. 1. It shows an analysis of the vertical input (unevennesses) $z_{1}-z_{4}$ of the four wheels $1-4$ of the model into input $z_{k s_{1}}, z_{k s_{2}}$ of the heave-pitch model [4], which will not be dealt with here, and input $z_{k p_{1}}, z_{k p_{2}}$ of the fourwheel roll model. This input will be marked $z_{k_{1}}, z_{k_{2}}$. Indices 1, 2 relate to front and rear axles. The mechanical schema of this roll-yaw model is shown in Fig. 2 .

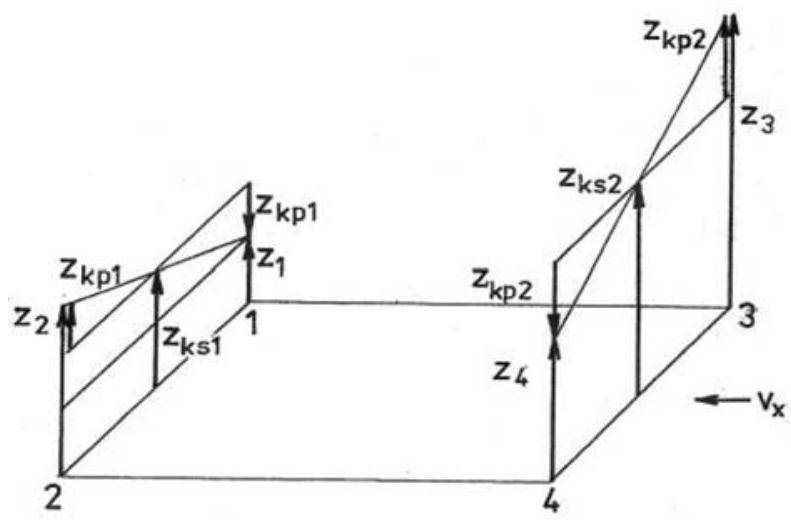

Fig. 1: Analysis of vertical inputs

This figure shows a model of a suspension with a solid rear axle. We will consider the radii of gyration of the body $r_{b x}$, of the seat $r_{s x}$, and of the axle $r_{w x}$. (The mass of the axle is assumed to be concentrated in the wheels.) Then there are the heights of the mass centers of the body $z_{t b}$, of the seat $z_{t s}$ and of the wheel $z_{t w}$. The track is denoted by $y_{w}$, the lateral distance of the seats by $y_{s}$, and the distance of the spring settings by $y_{c}$. The height of the joint of the solid axle is marked by $z_{q}$, and the lateral stiffness is marked by $k_{y}$. The lateral displacements of the wheels at stiffnesses $k_{y_{1}}, k_{y_{2}}$ are denoted by $Y_{k_{y_{1}}}$, $Y_{k_{y_{2}}}$. Vertical antiphased displacements of the body (above the wheel), seat and wheel are noted by $Z_{b}$, $Z_{s}$, and $Z_{w_{1}}, Z_{w_{2}}$. 


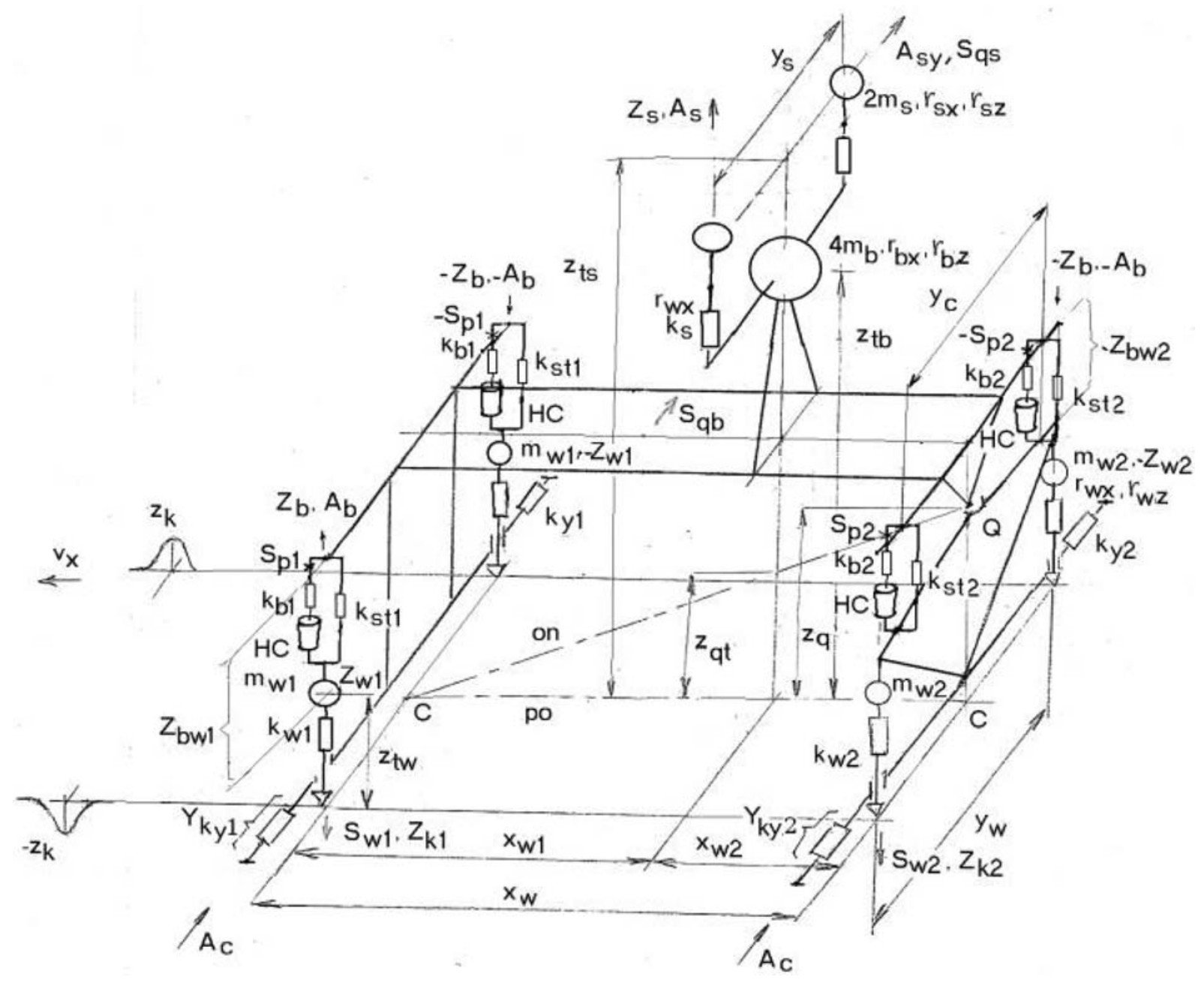

Fig. 2: Mechanical scheme

The most important input of this model is the lateral acceleration $A_{c}$.

We will take into account the lateral sliding of the rolling tyre. Sliding acts similarly to damper. Its damping is proportional to the weight of the vehicle and reciprocally proportional to the travelling velocity and the constant of the tyre $\operatorname{tg} \alpha$. The stiffness of a damper is its damping multiplied by operator $s$. So

$$
k_{y p k}=\mathbf{s} \cdot a_{g}\left(m_{b}+m_{s}+m_{w}\right) / v_{x} / \operatorname{tg} \alpha
$$

The lateral stiffness $k_{y}$ consists of this sliding stiffness $k_{y p k}$ and the lateral stiffness of the tyre $k_{w y}$ acting in series. So

$$
1 / k_{y}=1 / k_{w y}+1 / k_{y p k},
$$

We will use

$$
k_{w y r e}=0.6 k_{w}, \quad \operatorname{tg} \alpha=0.1 .
$$

The lateral input of the model is due to the lateral acceleration $A_{c}$ of the body.
The solid axle rotates around the center of the anti-phased unevennesses $C$, the body rotates around joint $Q$ and translates in lateral direction with the lateral displacement of the joint.

Only small angular displacements are assumed.

The lateral stiffness in front and rear, and also the wheel masses front and rear correspond to the position of the mass centre, i.e.

$$
k_{y_{1}} / k_{y_{2}}=m_{w_{1}} / m_{w_{2}}=x_{w_{2}} / x_{w_{1}}
$$

The roll model rotates around the on-axis, but its angle $\alpha$

$$
\alpha=\operatorname{arctg}\left(z_{q} / x_{w}\right)
$$

is small and approximately taken as $\cos \alpha=1$. Nevertheless, the efficient value of the axle joint height under the mass centre of the body is

$$
z_{q t}=x_{w_{1}} / x_{w} \cdot z_{q}
$$




\section{The equations of the mechanical model}

Two auxiliary constants are used to enable the equations to be used also for independent suspension, namely $t n=1, n z=0$ when with solid axle, $t n=0$, $n z=1$ when no solid axle is used. (With independent suspension the inertia forces of the wheel are supported by the body.)

If $Y z_{1}, Y z_{2}$ are the lateral displacements of the yaw motion, then the lateral force originating from the body mass $m_{b}$ is due to the acceleration of cornering, the roll movement of the body, the roll of the joint, and the lateral shift of the mass center, so

$$
\begin{aligned}
S_{q b}= & 4 m_{b} A_{c}+ \\
& 4 m_{b} \mathbf{s}^{2}\left[\left(z_{t b}-z_{q t}\right) Z_{b}+z_{q t} Z_{w_{2}}\right] \cdot 2 / y_{w}- \\
& 4 m_{b} \cdot s^{2} \cdot\left(x_{w_{2}} Y_{k y_{1}}+x_{w_{1}} Y_{k y_{2}}\right) / x_{w}
\end{aligned}
$$

and the lateral force of the seat

$$
\begin{aligned}
S_{q s}= & 4 m_{s} A_{c}+ \\
& 4 m_{s} \mathbf{s}^{2}\left[\left(z_{t s}-z_{q t}\right) Z_{b}+z_{q t} Z_{w_{2}}\right] \cdot 2 / y_{w}- \\
& 4 m_{s} \cdot s^{2} \cdot\left(x_{w_{2}} Y_{k y_{1}}+x_{w_{1}} Y_{k y_{2}}\right) / x_{w}
\end{aligned}
$$

The vertical forces in the suspension between body and wheel, front and rear

$$
\begin{aligned}
S_{b w_{1}}= & k_{b_{1}}\left(Z_{b}-Z_{w} !-Z_{r_{1}}\right)+ \\
& k_{s t_{1}}\left(Z_{b}-Z_{w} !\right) \\
S_{b w_{2}}= & k_{b_{2}}\left(y_{c} / y_{w} \cdot\left(Z_{b}-Z_{w_{2}}\right)-Z_{r_{2}}\right)+ \\
& k_{s t_{2}} y_{c} / y_{w} \cdot\left(Z_{b}-Z_{w_{2}}\right)
\end{aligned}
$$

Now the equation of moments to the body around the on-axis: tres

moments of the weight of the displaced mass cen-

$$
0=-8\left[\left(z_{t b}-z_{q t}\right) m_{b}+\left(z_{t s}-z_{q t}\right) m_{s}\right] a_{g} / y_{w} Z_{b}+
$$

moment of the inertia force from the roll motion

$$
\left.+8 \mathbf{s}^{2} m_{b} r_{b x}^{2}+m_{s} r_{s x}^{2}\right] / y_{w} \cdot Z_{b}
$$

moments of the forces in the suspension

$$
+y_{w} S_{b w_{1}}+y_{c} S_{b w_{2}}
$$

moment from the suspension of the seat

$$
-2 y_{s} k_{s}\left(Z_{s}-y_{s} Z_{b} / y_{w}\right)
$$

moments from the lateral forces

$$
+\left(z_{t b}-z_{q t}\right) S_{q b}+\left(z_{t s}-z_{q t}\right) S_{q s}
$$

moments of the roll motion of the wheels with independent suspension

$$
\begin{aligned}
& +2 \cdot\left(m_{w_{1}}+n z m_{w_{2}}\right)\left[2 \mathbf{s}^{2}\left(z_{t w}^{2}+r_{w}^{2}\right) / y_{w} \cdot Z_{b}-\right. \\
& \left.2 z_{t w} a_{g} / y_{w} \cdot Z_{b}\right]
\end{aligned}
$$

moments from the lateral motion of the wheels

$$
\begin{gathered}
+2 z_{t w}\left[\left(m_{w_{1}}+n z m_{w_{2}}\right) A_{c}-\right. \\
\left.s^{2}\left(m_{w_{1}} Y_{k y_{1}}+n z m_{w_{2}} Y_{k y_{2}}\right)\right]
\end{gathered}
$$

Equation of the forces vertical to the seat

$$
0=\mathbf{s}^{2} m_{s} Z_{s}+k_{s}\left(Z_{s}-y_{s} / y_{w} \cdot Z_{b}\right)
$$

Equation of vertical forces to the front wheels

$$
0=\mathbf{s}^{2} m_{w_{1}} Z_{w_{1}}+k_{w_{1}}\left(Z_{w_{1}}-Z_{k_{1}}\right)-S_{b w_{1}}
$$

Equation of moments to the solid rear axle:

From the inertia of the axle

$$
\begin{aligned}
& 0=2 \mathbf{s}^{2} m_{w_{2}}\left(y_{w}^{2} / 4+t n \cdot z_{t w}^{2}+t n \cdot r_{w x}^{2}\right) \\
& 2 / y_{w} \cdot Z_{w_{2}}
\end{aligned}
$$

from the unevennesses

$$
+y_{w} k_{w_{2}}\left(Z_{w_{2}}-Z_{k_{2}}\right)
$$

from the suspension

$$
-y_{c} S_{b w_{2}}
$$

from the lateral forces in the joint $S_{q b}, S_{q s}$

$$
+z_{q} x_{w_{1}} / x_{w} \cdot\left(S_{q b}+S_{q s}\right)
$$

from the lateral motion of the axle

$$
-2 t n \cdot m_{w_{2}}\left(z_{t w}-z_{q}\right)\left(A_{c}-s^{2} \cdot Y_{k y_{2}}\right)
$$

from the weight of the displaced mass centre

$$
-2 t n \cdot m_{w_{2}} z_{t w} \cdot 2 a_{g} Z_{w_{2}} / y_{w}
$$

from the weight of the body and seat to the displaced axle

$$
+8\left(m_{b}+m_{s}\right) a_{g} z_{q} Z_{w_{2}} / y_{w} \cdot x_{w_{1}} / x_{w}
$$

Equation of lateral forces

Lateral forces between the wheels and the road

$$
0=-2 k_{y_{1}} Y_{k y_{1}}-2 k_{y_{2}} Y_{k y_{2}}
$$

inertia forces of the body and seat

$$
+S_{q b}+S_{q s}
$$

from solid axle roll

$$
+s^{2} \cdot m_{w_{2}} \cdot z_{t w} \cdot 2 / y_{w} \cdot\left(t n \cdot Z_{w_{2}}+n z \cdot Z_{b}\right)
$$


from the lateral shift of the wheels

$$
\begin{aligned}
& +2\left(m_{w_{1}}+m_{w_{2}}\right) A_{c}- \\
& s^{2} \cdot\left(m_{w_{1}} Y_{k y_{1}}+m_{w_{2}} Y_{k y_{2}}\right)
\end{aligned}
$$

Finally, the equation of moments to the body around the $z$-axis passing through the center of gravity of the body: inertia moments of the body and seat

$$
\begin{aligned}
0= & s^{2}\left(4 m_{b} r_{b z}^{2}+4 m_{s} r_{s z}^{2}\right) \\
& \left(Y_{k y_{1}}-Y_{k y_{2}}-z_{q} \cdot 2 / y_{w} \cdot Z_{w_{2}}\right) / x_{w}
\end{aligned}
$$

plus the inertia moments of the wheels

$$
\begin{aligned}
& +s^{2}\left[2 m_{w_{1}}\left(r_{w z}^{2}+x_{w_{1}}^{2}+y_{w}^{2} / 4\right)+\right. \\
& \left.2 m_{w_{2}}\left(r_{w z}^{2}+x_{w_{2}}^{2}+y_{w}^{2} / 4\right)\right] \cdot \\
& \left(Y_{k y_{1}}-Y_{k y_{2}}-z_{q} \cdot 2 / y_{w} \cdot Z_{w_{2}}\right) / x_{w}
\end{aligned}
$$

and the moment of the forces between the wheels and the road

$$
+2 x_{w_{1}} k_{y_{1}} Y_{k y_{1}}-2 x_{w_{2}} k_{y_{2}}\left(Y_{k y_{2}}+z_{q} \cdot 2 / y_{w} \cdot Z_{w_{2}}\right)
$$

\section{The control}

Fig. 3 shows the control scheme of the suspension. In it, sensors are marked by their sensitivity constants.

We will assume that active suspension will be used only on the rear axle.
On the left side is the scheme of the front axle, with its spring $k_{b_{1}}$ and the damping $b_{b_{1}}$ and with static level control consisting of sensor $f_{z}$, very low pass VLP, hydraulic valve $V$, central accumulator $C A$ and drain $D R$. The dynamics of this control will be not dealt with here, i.e. $Z_{r_{1}}=0$.

Some explanation at first.

Elements marked SP are pressure sensors. A ring with minus in it produces the difference of two signals.

A ring with $N$ in it means a negator - an element converting the phase by $180 \mathrm{deg}$.

A ring with $P$ in it means an amplifier, with amplification proportional to the control input marked by an arrow.

Hydraulic linkage is shown by double lines, and the signal paths are shown by single lines. The directions of the signals are marked by triangular arrows. No feed-back through them is assumed. Signals coming to a common point add together. Crossing signal paths are not connected.

On the left side, the equipment of front wheels 1,2 is shown. On the right side, the equipment of rear wheels 3, 4 is shown. For reasons of stability, active suspension can be used only on one axle - let it be the rear axle. (Cars with front drive can use the front axle.) The lateral acceleration meter (with sensitivity constant $t_{a r}$ ) is mounted on the body at a height of $z_{t a}$.

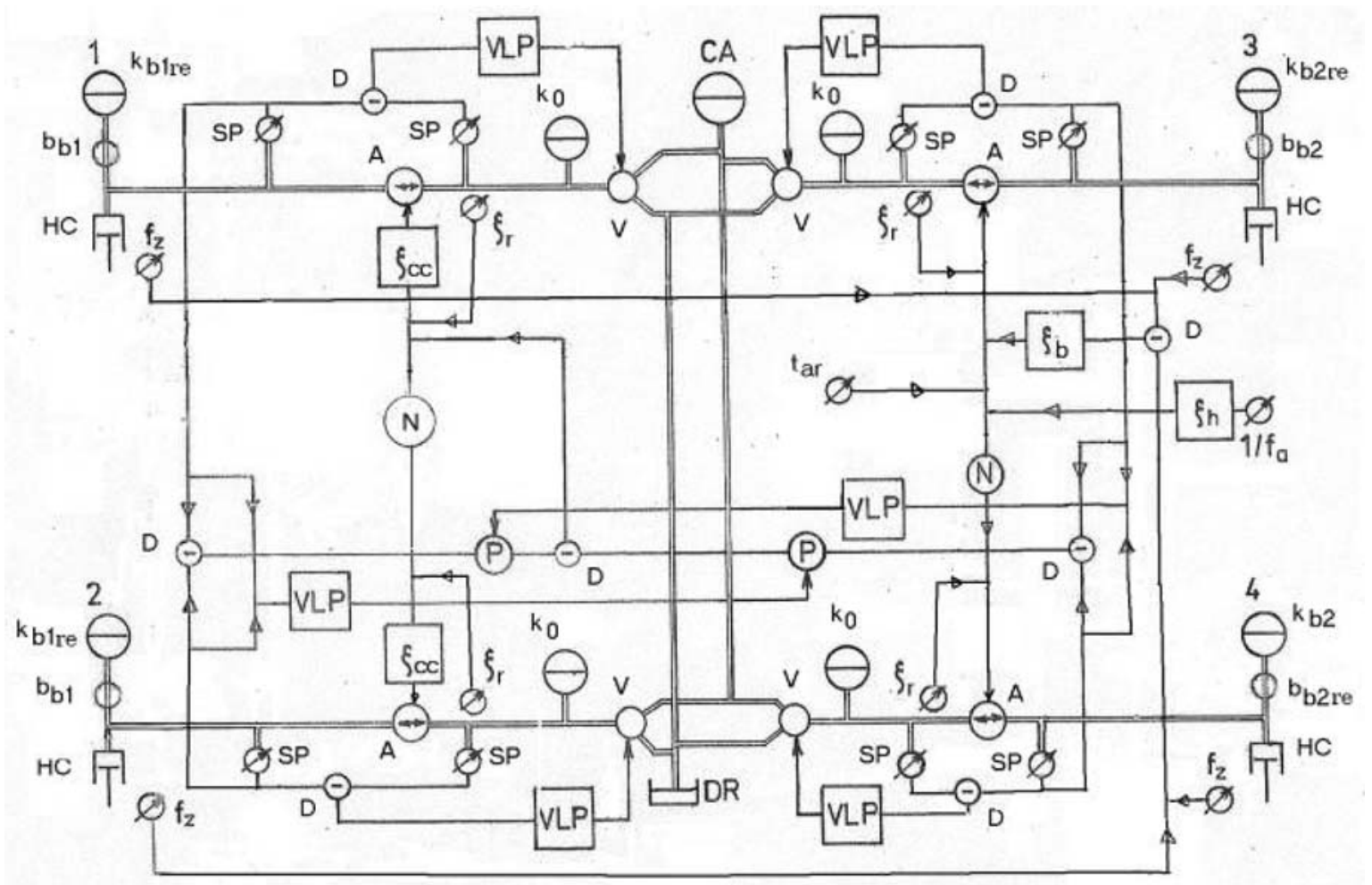

Fig. 3: Control scheme 
The active suspension on the rear axle (right part of the figure) consists of the sensors of the displacement wheel-body marked by $f_{z}$, producing the mean value of the front and rear displacements, and of the sensor of the vertical acceleration, marked by $1 / f_{a}$, and of the actuator embodied by a pump driven by an electric motor. The actuator is marked $A$, and its control amplification is $\zeta_{2}$, the common amplication of signals is $\zeta$. There is also a control velocity sensor, marked by its constant $\zeta_{r}$, to develop a source of velocity by means of a negative feedback. Thus, the force of the actuator (proportional to the mass $\left.m_{b}+m_{s}\right)$ is

$$
\begin{aligned}
S_{a}= & \zeta_{2} \mathbf{s} \zeta_{r} \cdot Z_{r_{2}}+\zeta \zeta_{2} \zeta_{d}\left[\zeta_{h} \cdot \mathbf{s}^{2} /\left(2 \pi f_{a}\right) \cdot Z_{b}+\right. \\
& 2 \pi f_{z} \zeta_{b}\left(Z_{b}-Z_{w_{1}} / 2-Z_{w_{2}} / 2\right)- \\
& \left(A_{c}-\mathbf{s}^{2} Y_{k y_{2}}+2 \mathbf{s}^{2}\left(\left(z_{t a}-z_{q t}\right) Z_{b}+\right.\right. \\
& \left.\left.\left.z_{q t} Z_{w_{2}}\right) / y_{w}-2 a_{g} Z_{b} / y_{w}\right) t_{a r}\right] b_{0} \\
& \left(m_{b}+m_{s}\right) / m_{0}
\end{aligned}
$$

where $b_{0}$ is a formal constant valued $1 \mathrm{~N} / \mathrm{ms}$ and $m_{0}$ is a constant with dimension $\mathrm{kg}$. and where suitable damping with damping coefficient $\beta_{b}$ is introduced by the element, marked by its transmission $\zeta_{b}=1+\mathbf{s} \cdot 2 \beta_{b} /\left(2 \pi f_{z}\right)$

Two filters are also included: a low pass with transmission $\zeta_{d}$ and cut-off frequency $f_{d}$, which helps to prevent undamping of the wheel, and a high-pass with transmission $\zeta_{h}$ and cut-off frequency $f_{h}$, which eliminates the false signal of the acceleration sensor of the tilted body. Namely

$$
\zeta_{d}=1 /\left(1+\mathbf{s} /\left(2 \pi f_{d}\right)\right), \quad \zeta_{h}=\mathbf{s} /\left(1+\mathbf{s} /\left(2 \pi f_{h}\right)\right)
$$

To the negative feedback: This control equation makes the actuator approximately a source of hydraulic current, i.e. the control displacement is not dependent on the force $S_{b w_{2}}$. (This can also be achieved by very high inner stiffness of the actuator, but with great losses of power.)

The force of the actuator is at the same time

$$
S_{r}=k_{r} Z_{r_{2}}+k_{0} Z_{r_{2}}-k_{b_{2}}\left(Z_{b_{2}}-Z_{w_{2}}-Z_{r_{2}}\right)
$$

where $Z_{r_{2}}$ is the control displacement and $k_{0}$ is the stiffness of the balancing spring in which the same static pressure is maintained as that in spring $k_{b}$ by means of sensors $S P$, differentiator $D$, very low pass $V L P$ and valve $V$. (In the computed examples $k_{0}=k_{b r e}$.) The inner stiffness of the actuator $k_{r}$ consists of the damping of pump $b_{r}$ and the inertia of the pump and the electric motor, so

$$
k_{r}=\mathbf{s} b_{r}+\mathbf{s} 2 m_{r}
$$

Combining equations 4.1 and 4.2 , a single control equation can be written, as follows

$$
\begin{aligned}
0= & \zeta_{2} \mathbf{s} \zeta_{r} Z_{r_{2}}+\zeta \zeta_{2} \zeta_{d}\left[\zeta_{h} \cdot \mathbf{s}^{2} /\left(2 \pi f_{a}\right) \cdot Z_{b}+\right. \\
& 2 \pi f_{z} \zeta_{b}\left(Z_{b}-Z_{w_{1}} / 2-Z_{w_{2}} / 2\right)- \\
& \left(A_{c}-\mathbf{s}^{2} Y_{k y}+2 \mathbf{s}^{2}\left(\left(z_{t a}-z_{q t}\right) Z_{b}+\right.\right. \\
& \left.\left.\left.z_{q t} Z_{w_{2}}\right) / y_{w}-2 a_{g} Z_{b} / y_{w}\right) t_{a r}\right]- \\
& {\left[\left(\mathbf{s} b_{r}+\mathbf{s}^{2} \cdot m_{r}\right) Z_{r}+k_{0} Z_{r}-\right.} \\
& \left.k_{b} \cdot\left(Z_{b}-Z_{w}-Z_{r}\right)\right] m_{0} /\left(m_{b}+m_{s}\right) / b_{0}
\end{aligned}
$$

Cross control is provided on the front axle. This cross control uses the measurements of the pressure transducers, marked $S P$ (in Fig. 3). The sum of the loads in the rear (after filtering through a very low pass $V L P$ to get static values $s_{s t_{1}}, s_{s t_{2}}$ ) controls the amplification of the proportional amplifier $P$ of the load difference of the front wheel load. The outputs of the two proportional amplifiers are compared, and their difference controls the pumps of the front wheels with the appropriate phase.

An equation to fulfil this aim can be written as follows

$$
\begin{aligned}
0= & \mathbf{s} Z_{r_{1}}+\zeta_{d p} v_{c c}\left[k _ { b r e _ { 2 } } \left(Z_{b}+\right.\right. \\
& \left.y_{w} / y_{c} \cdot Z_{r_{2}}-Z_{w_{2}}\right) /\left(x_{w_{1}} / x_{w}\right) / s_{s t_{1}}- \\
& \left.k_{b r e_{1}}\left(Z_{b}+Z_{r_{1}}-Z_{w_{1}}\right) /\left(x_{w_{2}} / x_{w}\right) / s_{s t_{2}}\right]
\end{aligned}
$$

where $v_{c c}$ is the control constant and where the transmission of the low path

$$
\zeta_{d p}=2 \pi f_{d p} /\left(2 \pi f_{d}+\mathbf{s}\right)
$$

with the characteristic frequency $f_{d p}$ delays the answer to antiphased unevennesses. The influence of the inner stiffness of the actuator is not included here (a source of displacement is assumed).

Equations 3.1 to $3.10,4.3,4.4$ make a set of equations for unknown quantities $S_{b w_{1}}, S_{b w_{2}}, S_{q b}, S_{q s}, Z_{b}$, $Z_{s}, Z_{w_{1}}, Z_{w_{2}}, Y_{k y_{1}}, Y_{k y_{2}}, Z_{r_{1}}, Z_{r_{2}}$.

\section{Input and output}

The spectrum of a surface of medium roughness is used for the statistical form of the road unevennesses (ride velocity $v_{x}=60 \mathrm{~km} / \mathrm{h}$ ):

$$
\begin{aligned}
Z_{f k}= & \sqrt{ }\left(v_{x}\right) / f \cdot \sqrt{ }(1 / 2- \\
& 1 / 2 /\left(1+\left(2 \pi \cdot f / 4.5 \cdot y_{w} / v_{x}^{4}\right)\right) / \\
& \left(1+\left(r_{p n} \cdot f / v_{x}\right) 2\right) \cdot 0.64 \mathrm{~mm} .
\end{aligned}
$$

where the track $y_{w}=1.8 \mathrm{~m}$. A correction to the tire diameter $r_{p n}$ is added. The effective value of this spectrum is $7.7 \mathrm{~mm}$.

The impulse input of the shape 1-cos (also "translated sinusoidal") was used in its spectral form

$$
\begin{aligned}
\tau f= & \sin \left(2 \pi f t_{i}\right) /(2 \pi f)+ \\
& t_{i} / 2 \cdot\left[\sin \left(\tau_{1}\right) / \tau_{1}+\sin \left(\tau_{2}\right) / \tau_{2}\right],
\end{aligned}
$$


where

$$
\tau_{1}=\pi-2 \pi f t_{i}, \quad \tau_{2}=\pi+2 \pi f t_{i}
$$

and where $t_{i}$ is the duration of the impulse at its half-height.

The maximum value of the impulse unevenness $z_{k i}$ is assumed to be proportional to its duration (progressive impulses).

These inputs are shown in the graphs of $Z_{f b w}$ or $Z_{b w}$.

The lateral input (lateral acceleration) is a sort of trapezoid-shaped impulse, but with rounded edges. It has been obtained by adding the two abovementioned curves $1-\cos$ with $t_{i} / 2$ (double cosinus), the second delayed by $t_{i}$ from the first.

This input will be attached to the graphs of lateral seat acceleration.

The graphs of the frequency characteristics $H_{f}$ have the effective values attached. These effective values follow the formula

$$
\sqrt{2 \int H_{f}^{2} \mathrm{~d} f} \text { within limits } 0: f_{\max }
$$

The pulse-effective values, given with time histories $h_{t}$, are the effective values of the whole answer divided by the pulse duration, ie.

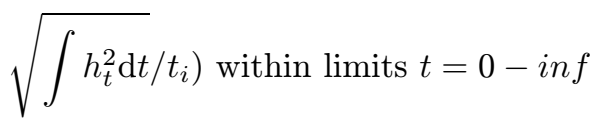

The vertical statistical input is due to the central path between the wheels.

The time interval between the front and the rear of the vertical input depends on the axle base $x_{w}$ and riding speed $v_{x}$

$$
t_{x}=x_{w} / v_{x} .
$$

So the relation of the vertical input front and rear is

$$
Z_{k_{2}}=Z_{k_{1}}\left(\cos \left(\mathbf{s} t_{x}\right)+i \sin \left(\mathbf{s} t_{x}\right)\right.
$$

\section{Criteria}

Lateral acceleration in the rear seat

$$
\begin{aligned}
A_{s y_{2}}= & A_{c}-\mathbf{s}^{2} \cdot Y_{k y_{2}}+ \\
& \mathbf{s}^{2}\left[\left(z_{t b}-z_{q t}\right) Z_{b}+z_{q} Z_{w_{2}}\right] \cdot 2 / y_{w}- \\
& 2 a_{g} Z_{b} / y_{w}
\end{aligned}
$$

Vertical acceleration in the seat

$$
A_{s}=\mathbf{s}^{2} Z_{s}
$$

Vertical acceleration of the body at radius $y_{w} / 2 y_{w} / 2$

$$
A_{b}=\mathbf{s}^{2} Z_{b}
$$

Body-wheel displacement front and rear

$$
\begin{aligned}
& Z_{b w_{1}}=Z_{b}-Z_{w_{1}}, \\
& Z_{b w_{2}}=Z_{b}-Z_{w_{2}} .
\end{aligned}
$$

Load transfer ratio front and rear

$$
\begin{aligned}
& S_{w_{1}} / s_{s t_{1}}=k_{w_{1}}\left(Z_{w_{1}}-Z_{k_{1}}\right) / s_{s t_{1}} \\
& S_{w_{2}} / s_{s t_{2}}=k_{w_{2}}\left(Z_{w_{2}}-Z_{k_{2}}\right) / s_{s t_{2}} .
\end{aligned}
$$

where the static forces are

$$
\begin{aligned}
& s_{s t_{1}}=2\left(m_{b}+m_{s}+m_{w}\right) a_{g} x_{w_{2}} / x_{w}, \\
& s_{s t_{2}}=2\left(m_{b}+m_{s}+m_{w}\right) a_{g} x_{w_{1}} / x_{w}
\end{aligned}
$$

Seat-body displacements front and rear

$$
\begin{aligned}
& Z_{s b_{1}}=Z_{s_{1}}-Z_{b}, \\
& Z_{s b_{2}}=Z_{s_{2}}-Z_{b}
\end{aligned}
$$

Dynamic-to-static ratio of lateral forces between wheels and road, front and rear

$$
\begin{aligned}
& S_{y_{1}} / s_{s t_{1}}=k_{y_{1}} Y_{k y_{1}} / s_{s t_{1}}, \\
& S_{y_{2}} / s_{s t_{2}}=k_{y_{2}} Y_{k y_{2}} / s_{s t_{2}} .
\end{aligned}
$$

Spring displacements, front and rear

$$
\begin{aligned}
& Z_{p_{1}}=Z_{b}-Z_{w_{1}}-Z_{r_{1}} \\
& Z_{p_{2}}=\left(Z_{b}-Z_{w_{2}}\right) y_{w} / y_{c}-Z_{r_{2}}
\end{aligned}
$$

The forces in the suspension, front and rear, are

$$
\begin{aligned}
& S_{b w_{1}}=k_{b_{1}}\left(Z_{b_{1}}-Z_{w_{1}}\right) \\
& S_{b w_{2}}=k_{b_{2}}\left(Z_{b_{2}}-Z_{w_{2}}-Z_{r_{2}}\right)
\end{aligned}
$$

\section{Universal parameters and used parameters}

The universal parameters (natural frequencies, damping coefficients) defined for the heave-pitch model are also used in the roll model, but we must also consider the anti-roll bar.

If the anti-roll bar stiffness-to-spring-stiffness $k_{b}$ ratio $\kappa_{s t a b}$ is given, then

$$
\begin{aligned}
k_{b}= & k_{b r e}+\mathbf{s} b_{b}=4 \pi^{2} f_{b}^{2}\left(m_{b}+m_{s}\right)\left(1+\kappa_{s t a b}\right)+ \\
& \mathbf{s} \cdot 4 \pi \beta_{b} f_{b}\left(m_{b}+m_{s}\right)
\end{aligned}
$$

Parameters used with a high bus $V$ variant $X-A$, i.e. cross control front and active control rear 


$$
\begin{array}{lllllll} 
& f_{b}=1 \mathrm{~Hz} & \beta_{b}=0.4 & m_{b}=1000 \mathrm{~kg} & z_{t b}=0.9 \mathrm{~m} & r_{b x}=0.6 \mathrm{~m} & y_{w}=1.8 \mathrm{~m} \\
& f_{w}=10 \mathrm{~Hz} & \beta_{w}=0.05 & m_{w}=150 \mathrm{~kg} & z_{t w}=0.5 \mathrm{~m} & r_{w x}=0.3 \mathrm{~m} & y_{c}=1.2 \mathrm{~m} \\
& f_{s}=3 \mathrm{~Hz} & \beta_{s}=0.28 & m_{s} / m_{b}=0.5 & z_{t s}=2.7 \mathrm{~m} & r_{s x}=0.4 \mathrm{~m} & y_{s}=1.2 \mathrm{~m} \\
\zeta=3 & f_{z}=0.4 \mathrm{~Hz} & \beta_{a}=1 & f_{a}=0.3 \mathrm{~Hz} & f_{d}=1 \mathrm{~Hz} & f_{h}=0.1 \mathrm{~Hz} & t_{a r}=0.075 \mathrm{~s} \\
\zeta_{2}=100 & \zeta_{r}=0.9 b_{r}=b_{b} / 1000 m_{r}=m_{b} / 1000 & b_{0}=1 \mathrm{Ns} / \mathrm{m} & m_{0}=1 \mathrm{~kg} & z_{t a}=0.9 \mathrm{~m} \\
& v_{c c}=1 \mathrm{~m} / \mathrm{s} \quad f_{c c}=3 \mathrm{~Hz} & \kappa_{s t a b_{1}}=0 & \kappa_{s t a b_{2}}=0 & z_{q}=0.3 \mathrm{~m} & x_{w}=5.5 \mathrm{~m} \\
x_{w_{1}} / x_{w_{2}}=2 \quad r_{b z}=2 \mathrm{~m} \quad r_{s z}=2 \mathrm{~m} \quad r_{w z}=0.3 \mathrm{~m} & &
\end{array}
$$
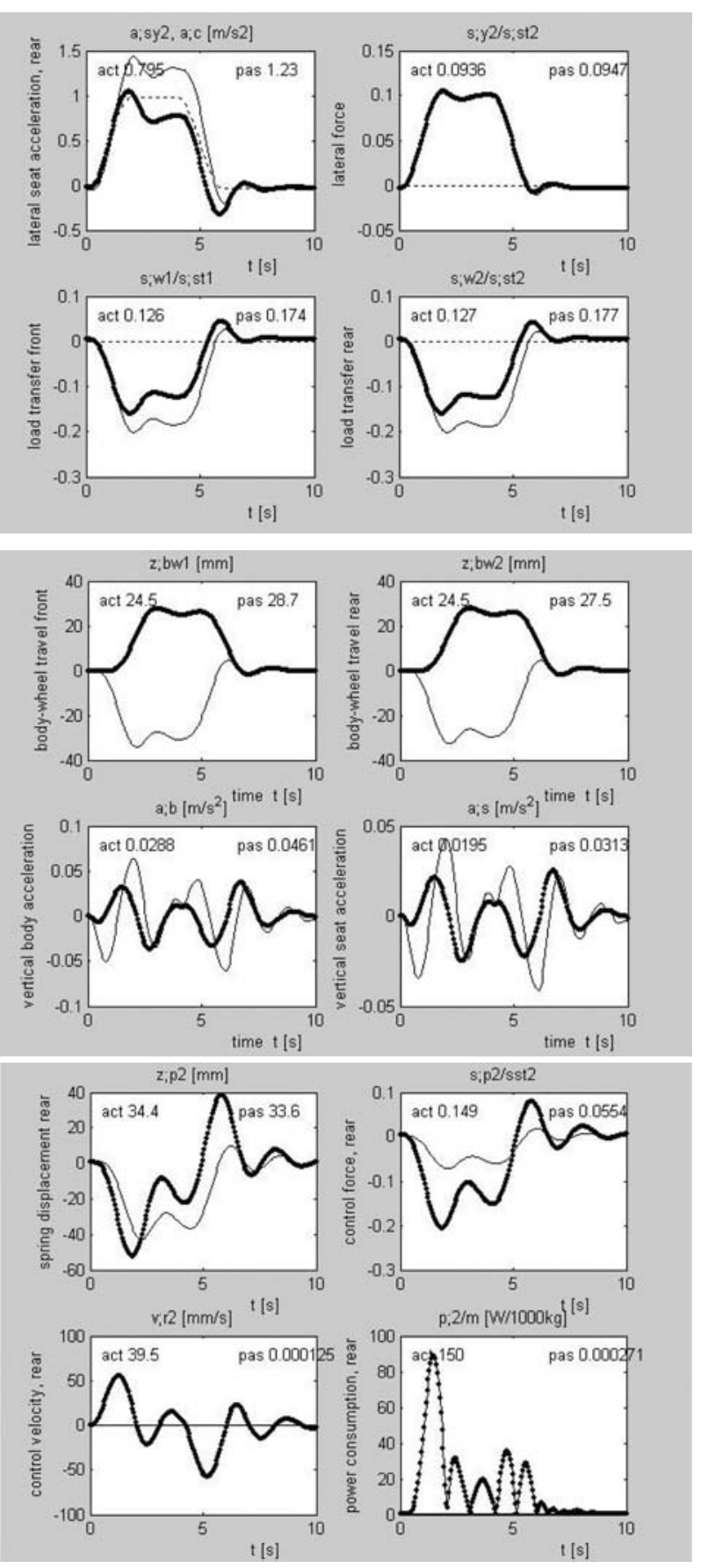

Fig. 4: Time histories due to the impulse of lateral acceleration. A thick line means an active variant (act), a thin line means a passive variant (pas). The attached numbers are imp-eff. values. A semicolon is used to mark an index. In the graph of power, the attached value refers to energy consumption [Ws/1000 kg]
Stiffnesses $k_{b}, k_{w}$ are assumed to be proportional to the static load front and rear:

$$
\begin{aligned}
& k_{b_{1}}=k_{b} \cdot 2 x_{w_{2}} / x_{w}, \quad k_{b_{2}}=k_{b} \cdot 2 x_{w_{1}} / x_{w}, \\
& k_{w_{1}}=k_{w} \cdot 2 x_{w_{2}} / x_{w}, \quad k_{w_{2}}=k_{w} \cdot 2 x_{w_{1}} / x_{w}
\end{aligned}
$$

For variant $P-P$, i.e. for a vehicle with passive suspension front and rear, the parameters differ as follows:

$$
\begin{aligned}
\kappa_{s t a b_{1}} & =1.2 & \kappa_{s t a b_{2}} & =1.4 \\
z_{q} & =0.9 \mathrm{~m} & \zeta_{2} & =100
\end{aligned}
$$

The parameters of actuators $m_{r}$ and $b_{r}$ are only estimated.

\section{Example of modelling results}

In the graphs in Figs. 4, 5, the active variant act (passive suspension front, active rear) is indicated by more prominent lines (lines with dots) than those of the passive variant (pas-pas). In some suitable graphs, the appropriate input is shown by interrupted lines.

Effective values (with frequency characteristics) or impulse-effective values (effective values of the answers divided by the impulse duration, with time histories) are attached to the labels.

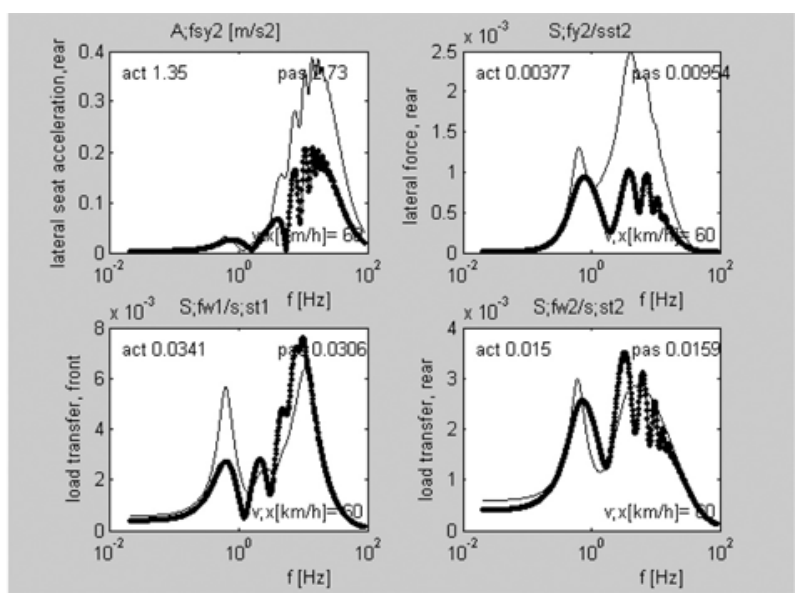

Fig. 5: Frequency characteristics due to unevennesses. A thick line means an active variant (act), a thin line means a passive variant (pas). The attached numbers are impeff. values. A semicolon is used to mark an index 
Steady-state values due to

Lateral acceleration in the seat

Body-wheel travel

Vertical dynamic force to static force ratio, front

Vertical dynamic force to static force ratio, rear

Steady-state values due to in-phase unevenness

Lateral acceleration in the seat

Vertical dynamic force to static force ratio, front

Vertical dynamic force to static force ratio, rear

Steady-state values due to cross unevenness

Lateral acceleration in the seat

Vertical dynamic force to static force ratio, front

Vertical dynamic force to static force ratio, rear

\section{$9 \quad$ Stability}

Stability against self-exciting oscillation is an important criterion of the system. It is most important with the roll model, where there is also the possibility of roll-over. Asymptotic stability is used, i.e. the real part of the eigenvalues of the equation matrix must be negative. Stability was checked for all parameters of a simplified model. The parameters of nominal value $h_{n o m}$ were varied between $1 / 100$ to 100 multiples of its given value $h_{j m}$. When no instability was found, output 100 was given. If instability was found at parameter value $h_{c r i t}$, the stability rate $h_{\text {crit }} / h_{\text {nom }}$ was put out. A minimal stability rate of about 2.2 of the passive model increases to 5 with active suspension.

\section{Conclusions based on the example}

With vertical statistical input, the lateral acceleration in the seat is slightly increased and the lateral force is substantially decreased. The lateral load transfer is slightly deteriorated, but the effective values incorporating the values from the heave-pitch model are little influenced. It has been shown that it is possible by means of cross control to distribute the load changes due to cornering proportional to the static load of the axle. The lateral input is the main advantage of active suspension, thanks to the active roll:

From the viewpoint of ride comfort, active roll substantially suppresses the lateral force during cornering. From the viewpoint of ride safety, active roll also substantially suppresses the load transfer to the outside wheels in a curve. Thus the vehicle can take

$\begin{array}{lll} & \text { active } & \text { passive } \\ a_{c}=1 \mathrm{~m} / \mathrm{s}^{2} & & \\ a_{s y}\left[\mathrm{~mm} / \mathrm{s}^{2}\right] & 0.77 & 1.35 \\ z_{b w_{2}}[\mathrm{~mm}] & 25 & 25 \\ s_{w_{1}} / s_{s t_{1}} & 12 & 20 \\ s_{w_{2}} / s_{s t_{2}} & 14 & 20 \\ z_{k_{1}}=z_{k_{2}}=0.01 \mathrm{~m} & \\ A_{s y}\left[\mathrm{~m} / \mathrm{s}^{2}\right] & 0.11 & 0.15 \\ S_{w_{1}} / s_{s t_{1}} & 0.02 & 0.022 \\ S_{w_{2}} / s_{s t_{2}} & 0.017 & 0.019 \\ z_{k_{1}}=0.01 \mathrm{~m}, & z_{k_{2}}=-0.01 \mathrm{~m} \\ A_{s y}\left[\mathrm{~m} / \mathrm{s}^{2}\right] & 0.028 & 0.023 \\ S_{w_{1}} / s_{s t_{1}} & 0.00042 & -0.07 \\ S_{w_{2}} / s_{s t_{2}} & 0.009 & 0.03\end{array}$

curves at a higher speed or with more safety.

With active roll suspension there is no need to use a solid axle for anti-roll purposes. This means more comfort for the passenger and there is reduced damage to the road, due to a big reduction in the lateral forces between wheel and road. A solid axle was used in our examples, but with reduced height of the roll axis.

With active suspension there is also no need for anti-roll bars. Active roll technology also enables the design of a slender passenger car for two passengers sitting in tandem (we can call this a tandemo) with, e.g., an $0.8 \mathrm{~m}$ track.

\section{References}

[1] Sampson, D. J. M., Cebon, D.: Active Roll Control of Single Unit Heavy Road Vehicles, http://www.cvdc.org/recent_papers/ SampsonCebon_VSD02.pdf

[2] Vaculín, O., Valášek, M., Svoboda, J.: Influence of Vehicle Tilting on Its Performance, Conf. Interaction and Feedbacks, Praha, 2005.

[3] Masashi, Yamashita: Application of H-Y Control to Active Suspension Systems, Automatica, Vol. 30, 1994.

[4] Čech, I.: A Pitch-Plane Model of a Vehicle with Controlled Suspension. Vehicle System Dynamics, Vol. 23, 1998, pp. 133-148.

Ing. Ilja Čech

E-mail: cech10600jes11@seznam.cz

Jesenická 11, 10600 Prague 10, Czech Republic 


\section{List of symbols}

\section{Complex amplitudes, spectral values and instantaneous values}

$A_{c} A_{f c} a_{c}$ $A_{s} A_{f s}$ $A_{s y} A_{f t y} a_{s y}$ $A_{x} A_{f x}$ $S_{a} S_{f a} s_{a}$

$S_{b w} S_{f b w} s_{b w}$

$S_{q} S_{f q} s_{q}$

$S_{p} S_{f p} s_{p}$

$S_{q b} S_{f q b} s_{q b}$

$S_{q s} S_{f q s} s_{q s}$

$S_{s b} S_{f s b} s_{s b}$

$S_{w} S_{f w} s_{w}$

$S_{y} S_{f y} s_{y}$

$V_{r} V_{f r} v_{r}$

$Y_{a r} Y_{f a r} y_{a r}$

$Y_{k y} Y_{f k y} y_{k y}$

$Z_{b} Z_{f b} z_{b}$

$Y_{z} Y_{f z} y_{z}$

$Z_{b w} Z_{f b w} z_{b w}$

$Z_{k} Z_{f k} z_{k}$

$Z_{r} Z_{f r} z_{r}$

$Z_{s} Z_{f s} z_{s}$

$Z_{w} Z_{f w} z_{w}$ lateral (centripetal) acceleration $\left[\mathrm{m} / \mathrm{s}^{2}\right]$

as vertical acceleration of the seat $\left[\mathrm{m} / \mathrm{s}^{2}\right]$

lateral acceleration in the seat above the center of gravity $\left[\mathrm{m} / \mathrm{s}^{2}\right]$

ax longitudinal acceleration input $\left[\mathrm{m} / \mathrm{s}^{2}\right]$

force developed by the actuator $[\mathrm{N}]$

force between body and wheel $[\mathrm{N}]$

lateral inertia force due to yaw motion $[\mathrm{N}]$

force on the spring $[\mathrm{N}]$

lateral force in the joint due to the body $[\mathrm{N}]$

lateral force in the joint due to the seat $[\mathrm{N}]$

vertical force between seat and body $[\mathrm{N}]$

vertical force between wheel and road surface $[\mathrm{N}]$

lateral force between wheel and road surface $[\mathrm{N}]$

velocity of movement of the actuator $[\mathrm{m} / \mathrm{s}$ ]

lateral displacement of the body at lateral acceleration meter height $[\mathrm{m}]$

lateral displacement in lateral stiffnesses $k_{y_{1}}, k_{y_{2}}$

vertical displacement (for the roll model at radius $y_{w} / 2$ ) $[\mathrm{m}]$

lateral displacement of the body due to yaw motion [m]

body-wheel displacement $[\mathrm{m}]$

vertical input displacement $[\mathrm{m}]$

control displacement $[\mathrm{m}]$

vertical seat displacement $[\mathrm{m}]$

vertical wheel displacement $[\mathrm{m}]$

\section{Quantities without physical dimension}

$\beta_{a}$

$\beta_{b}$

$\beta_{s}$

$\zeta$

$\zeta_{2}$

$\zeta_{b}$

$\zeta_{d}$

$\zeta_{h}$

$\zeta_{r}$

$\kappa_{\text {stab }}$

$\kappa_{y w}$

$n z, \operatorname{tn} n z=0, t n=1$ relative damping rate of the control

relative damping rate of the body

relative damping rate of the seat

common amplification of signals

amplification of the actuator

corrective damping element

transmission of low pass filter

transmission of high pass filter

sensitivity constant of feedback sensor

ani-roll bar stiffness

lateral stiffness to radial stiffness ratio

for solid axle, $n z=1, t n=0$ for no solid axle

\section{Other quantities}

$a_{g} \quad$ gravity acceleration $\left[\mathrm{m} / \mathrm{s}^{2}\right]$

$b_{b} \quad$ damping of passive suspension $[\mathrm{Ns} / \mathrm{m}]$

$b_{p k} \quad$ slip damping of the tyre $[\mathrm{Ns} / \mathrm{m}]$

$b_{0} \quad$ formal constant of the actuator $b_{0}=1[\mathrm{Ns} / \mathrm{m}]$

$b_{r} \quad$ damping of the actuator $[\mathrm{Ns} / \mathrm{m}]$

$b_{s} \quad$ seat damping $[\mathrm{Ns} / \mathrm{m}]$

$b_{w} \quad$ wheel damping $[\mathrm{Ns} / \mathrm{m}]$

$f \quad$ frequency $[\mathrm{Hz}]$

$f_{a} \quad 1 /(2 \pi f a)$ sensitivity constant of transducer of vertical acceleration $[\mathrm{Hz}]$

$f_{b} \quad$ natural frequency of passive suspension $[\mathrm{Hz}]$

$f_{d}, f_{h} \quad$ characteristic frequency of the filter $[\mathrm{Hz}]$ 
$f_{r} \quad$ sensitivity constant of the control velocity sensor $[\mathrm{Hz}]$

$f_{z} \quad 2 \pi f_{z}$ constant of the displacement transducer $[\mathrm{Hz}]$

$f_{\varepsilon} \quad 1 /\left(2 \pi f_{\varepsilon}\right)$ constant of the roll acceleration transducer $[\mathrm{Hz}]$

$h_{j} \quad$ nominal value of the parameter

$h_{k r i t} \quad$ critical value of the parameter

$k_{0} \quad$ spring rate of the balancing spring $[\mathrm{N} / \mathrm{m}]$

$k_{b} \quad$ stiffness of the body-wheel suspension $[\mathrm{N} / \mathrm{m}]$

$k_{\text {bre }} \quad$ spring rate of the body-wheel spring $[\mathrm{N} / \mathrm{m}]$

$k_{r} \quad$ inner stiffness of the actuator $[\mathrm{N} / \mathrm{m}]$

$k_{s} \quad$ stiffness of the seat-body spring $[\mathrm{N} / \mathrm{m}]$

$k_{s t} \quad$ stiffness of the roll-bar $[\mathrm{N} / \mathrm{m}]$

$k_{w} \quad$ radial stiffness of the tyre $[\mathrm{N} / \mathrm{m}]$

$k_{\text {wyre }} \quad$ real lateral stiffness of the tyre $[\mathrm{N} / \mathrm{m}]$

$k_{y} \quad$ lateral stiffness $[\mathrm{N} / \mathrm{m}]$

$k_{y p k} \quad$ sliding stiffness $[\mathrm{N} / \mathrm{m}]$

$m_{0} \quad$ formal constant $m_{0}=1 \mathrm{~kg}$

$m_{b} \quad$ quarter body mass $[\mathrm{kg}]$

$m_{s} \quad$ quarter seat mass $[\mathrm{kg}]$

$m_{c} \quad$ total mass of vehicle $m_{b}+m_{s}+m_{w}[\mathrm{~kg}]$

$m_{r} \quad$ actuator mass transferred to the pump radius [kg]

$m_{w} \quad$ wheel mass [kg]

$p \quad$ power $[\mathrm{W}]$

$r_{p n} \quad$ tyre radius $[\mathrm{m}]$

$r_{b x} \quad$ gyration radius of the body to the longitudinal axis [m]

$r_{b z} \quad$ gyration radius of the body to the vertical axis [m]

$r_{s x} \quad$ gyration radius of the seat to the longitudinal axis [m]

$r_{s z} \quad$ gyration radius of the seat to the vertical axis [m]

$r_{w x} \quad$ gyration radius of the wheel to the longitudinal axis [m]

$r_{w z} \quad$ gyration radius of the wheel to the vertical axis $[\mathrm{m}]$

s operator $i 2 \pi f$

$t \quad$ time $[\mathrm{s}]$

$t_{\text {ar }} \quad$ sensitivity constant of the lateral acceleration sensor $[\mathrm{Hz}]$

$t_{\text {gal }} \quad$ constant of inverse proportionality between slip rotation and load

$t_{i} \quad$ duration of input impulse at half-height $[\mathrm{s}]$

$v_{x} \quad$ travelling speed $[\mathrm{m} / \mathrm{s}]$

$y_{c} \quad$ spring distance $[\mathrm{m}]$

$y_{s} \quad$ seat distance $[\mathrm{m}]$

$y_{w} \quad$ wheel track $[\mathrm{m}]$

$z_{a} \quad$ height of the lateral acceleration meter $[\mathrm{m}]$

$z_{k i} \quad$ maximum value of the vertical input impulse $[\mathrm{m}]$

$z_{q} \quad$ height of the joint $[\mathrm{m}]$

$z_{q t} \quad$ height of the axis on under the center of gravity [m]

$z_{t a} \quad$ height of the lateral acceleration transducer $[\mathrm{m}]$

$x_{w} \quad$ axle base $[\mathrm{m}]$

$x_{w_{1}} \quad$ distance between the mass centre of the body and the front axle [m]

$x_{w_{2}} \quad$ distance between the mass centre of the body and the rear axle $[\mathrm{m}]$

$z_{t b} \quad$ height of the body mass centre [m]

$z_{t s} \quad$ height of the seat mass centre $[\mathrm{m}]$

$z_{t w} \quad$ height of the wheel mass centre $[\mathrm{m}]$

\section{Indices}

1 front axle

2 rear axle

$b \quad$ body

$f \quad$ spectral values

re real part $s \quad$ seat

$x \quad$ longitudinal direction, axis

$y \quad$ lateral direction, axis

$w \quad$ wheel 